\title{
CMS GEM detector material study for the HL-LHC
}

\section{S. Muhammad*}

University of Roma "La Sapienza", Rome, Italy; Laboratori Nazionali di Frascati - INFN,

Frascati, Italy; and National Centre for Physics, QAU, Islamabad, Pakistan; E-mail:

Saleh.Muhammadecern.ch

\section{G. Saviano}

University of Roma "La Sapienza", Rome, Italy; and Laboratori Nazionali di Frascati - INFN,

Frascati, Italy; E-mail: giovanna.saviano@uniromal.it

\section{S. Bianco, L.Benussi, D. Piccolo, G. Raffone, L. Passamonti, D. Pierluigi, A. Russo}

Laboratori Nazionali di Frascati - INFN, Frascati, Rome, Italy

\section{Valente, M. Ferrini, F. Sarasini, A. Lalli, A.V. Franchi, J. Tirilló}

University of Roma "La Sapienza", Department of Chemical Engineering Materials

Environment and UdR INSTM, Rome, Via Eudossiana, 18 00184, Rome, Italy

\section{Caponero}

Centro Ricerche ENEA Frascati, via E. Fermi 45, 00044 Frascati, Rome and Laboratori

Nazionali di Frascati - INFN, Frascati, Italy

\begin{abstract}
A study on the Gaseous Electron Multiplier (GEM) foil material is performed to determine the moisture diffusion rate, moisture saturation level and the effects on its mechanical properties. The study is focused on the foil contact with ambient air and moisture to determine the value of the diffusion coefficient of water in the foil material. The presence of water inside the detector foil can determine the changes in its mechanical and electrical properties. A simulated model is developed with COMSOL Multiphysics v. 4.3 [1] by taking into account the real GEM foil (hole dimensions, shapes and material), which describes the adsorption of water. This work describes the model, its experimental verification, the water diffusion within the entire sheet geometry of the GEM foil, thus gaining concentration profiles and the time required to saturate the system and the effects on the mechanical properties.
\end{abstract}

EPS-HEP 2017, European Physical Society conference on High Energy Physics

5-12 July 2017

Venice, Italy

\footnotetext{
*Speaker.
} 


\section{Introduction}

The Gaseous Electron Multiplier (GEM) detector has been approved for the upgrade of the forward muon system of Compact Muons Solenoid (CMS) at Large Hadron Collider (LHC). The GEM detector provides good timing and position resolution and it meets the particle detection requirements in the eta region $1.6<\eta<2.2$, where high particle flux $5 \mathrm{kHz} / \mathrm{cm}^{2}$ is expected [2] [3]. The principal component of the detector is a GEM foil $(\mathrm{Cu}$-cladded $(5 \mu \mathrm{m})$, micro perforated $60 \mu \mathrm{m}$-thick kapton film), that provides charge amplification thanks to the conical shape of very dense micro holes.
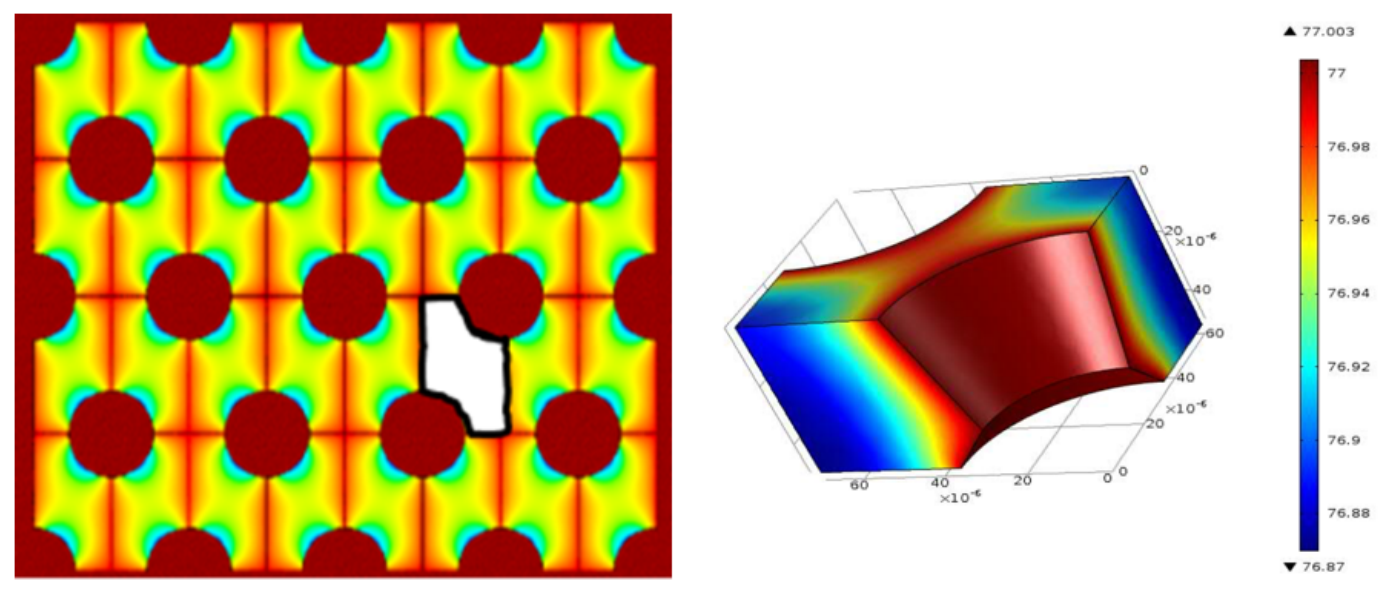

Figure 1: (left) Geometry of a GEM and detail analysis part is in black and white [5], (right). Concentration profile after 44400s with $\mathrm{RH}=4 \%$.

\section{Development of a diffusion model using COMSOL Multiphysics}

This study considers the diffusion of $\mathrm{H}_{2} \mathrm{O}$ from the hole surface only, since no penetration in polyimide is possible through $\mathrm{Cu}$ which behaves as a waterproof barrier. The presence of any stagnant $\mathrm{H}_{2} \mathrm{O}$ film around the surface of the hole is also neglected, since the gas is continuously flushed inside the chamber, thus allowing to always have a constant and uniform concentration on the surface of the hole, i.e., results represents the maximum concentration of water that can get inside the structure considered. In general, moisture comes from the surrounding environment and spreads within the polyimide. The relationship used to obtain the value of this concentration on the surface of a polyimide as a function of the external humidity is derived from the literature [6]

$$
c_{1}=4.520 \times 10^{-4} \times R H-8.319 \times 10^{-4}
$$

where $c_{1}$ expressed in grams of water to grams of polyimide. The formula used is empirical and it is compatible with the official data released by the manufacturer [7].

\section{Experimental setup for verification of the diffusion model}

The test apparatus setup in the Frascati INFN Lab consists of a weighing balance "Analytic balance Gibertini E42S", whose measurements are recorded by using a camera in front of the ana- 
lytical balance display and connected to a computer. A data acquisition program is used to record weight measurements with constant time intervals. The sample used for this test has dimensions of $105 \mathrm{~mm} \times 50 \mathrm{~mm} \times(50+5+5) \mu \mathrm{m}$, and it was previously conditioned in an oven at $(105 \pm 5)$ ${ }^{o} \mathrm{C}$ for 36 hours.
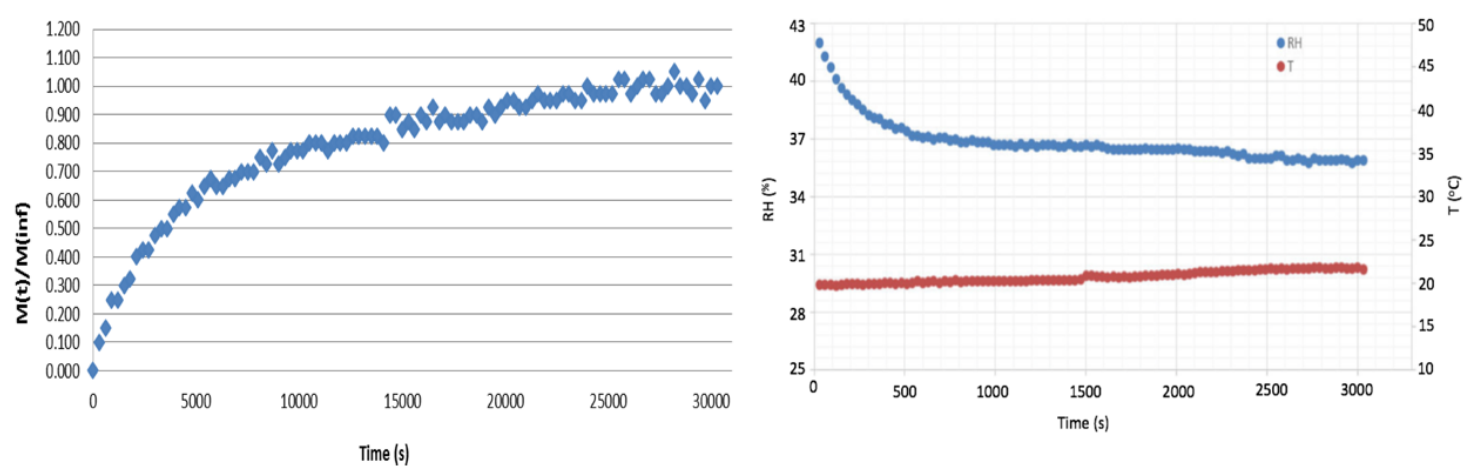

Figure 2: (left). Saturation trends of the GEM foil, (right). Temperature (T) and Relative Humidity (RH) in the container during the test.

\section{Moisture and dryness effects on the GEM and kapton tensile properties}

To dry GEM and kapton samples an oven at $99^{\circ} \mathrm{C}$ is used for 36 hours. Conditioning to a wet state is accomplished by keeping samples at $99.5 \%$ relative humidity and room temperature ( 25 ${ }^{o} \mathrm{C}$ ) for five days. Tensile tests were performed in displacement control using a crosshead speed of $10 \mathrm{~mm} / \mathrm{min}$ and a gauge length of $70 \mathrm{~mm}$ by means of a Zwick/Roell Z010 according to ASTM D882-02 standard. The measurements were performed at room temperature $\left(25^{\circ} \mathrm{C}\right)$ until final failure of the samples occurred. Each set such as GEM dry, GEM wet, kapton dry and kapton wet has four samples.
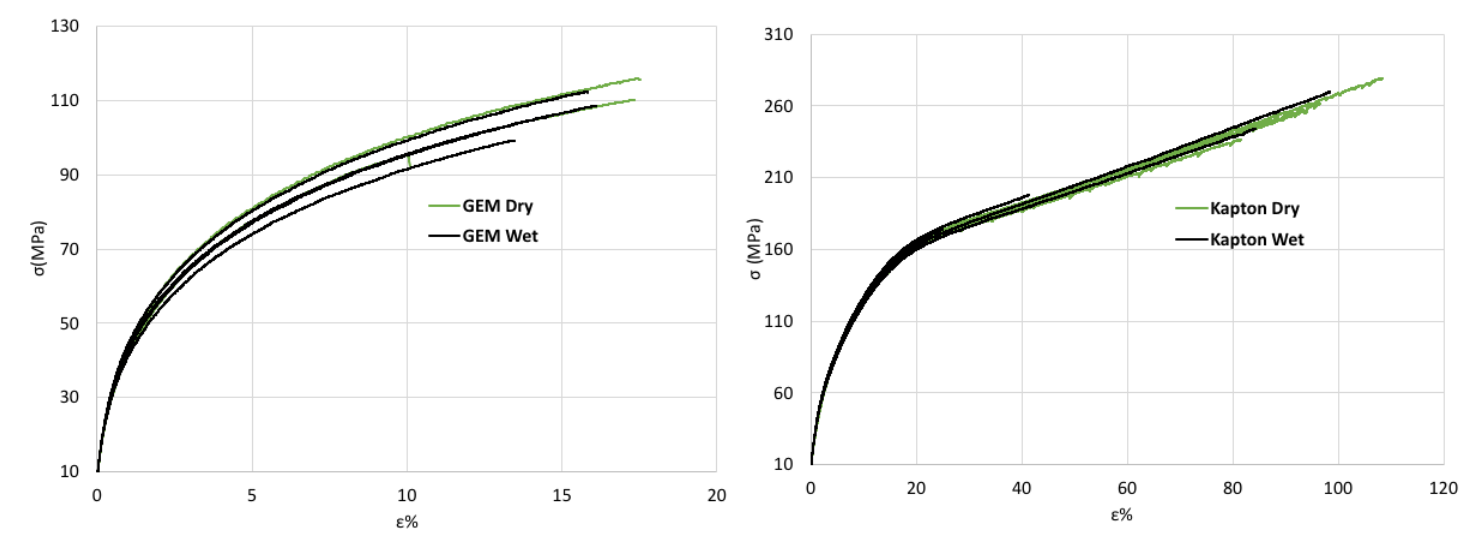

Figure 3: (left) Tensile stress vs elongation trends for kapton dry and wet, (right). Tensile stress vs elongation trends for GEM dry and wet. 
Table 1: Young's modulus of kapton and GEM foil

\begin{tabular}{|c||c||c|}
\hline \hline Condition & Kapton Young's modulus (MPa) & GEM Young's modulus (MPa) \\
\hline Dry & $2385 \pm 20$ & $6026 \pm 119$ \\
Wet & $2529 \pm 57$ & $6314 \pm 254$ \\
\hline
\end{tabular}

\section{Results discussion}

The simulation results showed that the saturation time is about 10 hours at $\mathrm{RH}=4 \%, 14$ hours at $\mathrm{RH}=40 \%$ and $100 \%$, starting from a null value of water inside. The concentrations limits are different $75 \mathrm{~mol} / \mathrm{m}^{3}, 1380 \mathrm{~mol} / \mathrm{m}^{3}$ and $3450 \mathrm{~mol} / \mathrm{m}^{3}$ at different relative humidity values $4 \%, 40$ $\%$ and $100 \%$ respectively [8]. In figure 1 , the geometry of a GEM and concentration profile after $44400 \mathrm{~s}$ with $\mathrm{RH}=4 \%$ is shown.

After the conditioning in oven, the sample is weighed and a value of $(0.6575 \pm 0.0002) \mathrm{g}$ is found, while the bolt placed on the scale plate had a weight of $5,725 \mathrm{mg}$. The humidity and temperature range during this test is selected as $\mathrm{RH}=34-36 \%$ and $\mathrm{T}=19-20^{\circ} \mathrm{C}$, the images are taken from every 5 minutes interval. The trends of saturation along with temperature and humidity during tests are shown in figure 2, showing how in 8-9 hours it is possible to saturate completely the GEM foil. These results confirm the model in [8]. Furthermore, the measurements showed the presence of $0.004 \mathrm{~g}$ water at saturation, comparable with what is obtained from the official data sheet by the manufacturer [7] or from equation 2.1, for a value of $\mathrm{RH}=37.5 \%$. By using the experimental data, we calculated the diffusion coefficient of water in the foil as $\mathrm{D}_{G E M}=(3.3 \pm 0.1$ (stat) $) 10^{-10} \mathrm{~cm}^{2} / \mathrm{s}[9]$.

Tensile trends of GEM and kapton are shown in figure 3. An average Ultimate Tensile Stress (UTS) and elongation values of the dry GEM are $106.0 \pm 12.2 \mathrm{MPa}$ and $14.9 \pm 4.2 \%$ respectively. Similarly, for the wet GEM the average UTS and elongation values are $106.3 \pm 5.3 \mathrm{MPa}$ and 14.9 $\pm 1.2 \%$ respectively. An average UTS and elongation of the four dry kapton samples is 258.2 $\pm 17.6 \mathrm{MPa}$ and $95 \pm 11 \%$, respectively and for the wet kapton the figures are $221.1 \pm 43.2$ MPa and $62 \pm 34 \%$, respectively. Measurements of UTS and elongation showed that within the experimental errors; the dry kapton is stronger than the wet kapton. For GEM foils it is shown how average UTS values see no difference between dry and wet. Young's modulus is estimated from stress vs strain data and is shown in table 1.

\section{References}

[1] COMSOL Multiphysics version 4.3, (C)1998-2013 by COMSOL, Inc.

[2] A. Colaleo et al., CMS TECHNICAL DESIGN REPORT FOR THE MUON ENDCAP GEM UPGRADE, CERN-LHCC-2015- 012. CMS-TDR- 013, <https://cds.cern.ch/record/2021453>.

[3] D. Abbaneo et al 2015 JINST 10 C03039, Quality control and beam test of GEM Detectors for future upgrades of the CMS muon high rate region at the LHC <doi:10.1088/1748-0221/10/03/C03039>.

[4] Sacher E., Susko; Water Permeation of Polymers Films. I. Polyimide, Material and Engineering Analysis, IBM Corporation System Division Endicott, New York; 1978.

[5] G.Raffone, CMS Trapezoidal GEM Foils Structural Analysis, preprint LNF-10/20(IR). 
[6] Sacher E., Susko; Water Permeation of Polymers Films. I. Polyimide, material and engineering analysis, IBM Corporation System Division Endicott, New York, 1978.

[7] Kapton-HN Technical Info; DuPont, <www.dupont.com/products-andservices/membranes-films/polyimide-films/brands/kapton-polyimide-film.htm>.

[8] Benussi, L. et al., Characterization of the GEM foil materials, 4th International Conference on Micro Pattern Gaseous Detectors (MPGD2015) Trieste, Italy, October 12-15, 2015, <http://inspirehep.net/record/1411755/files/arXiv:1512.08621.pdf>.

[9] A.V. Franchi et al., Test for the Measurement of Diffusion Coefficient of Water in Kapton Foils for the GEM Detector of the Upgraded High-Pseudorapidity Muon Detection in CMS, preprint INFN-13-09/LNF. 